\title{
Meditation and its regulatory role on sleep
}

\author{
Ravindra P. Nagendra, Nirmala Maruthai and Bindu M. Kutty* \\ Department of Neurophysiology, National Institute of Mental Health and Neurosciences, Bangalore, India
}

\author{
Edited by: \\ V. Mohan Kumar, Sree Chitra Tirunal \\ Institute for Medical Sciences and \\ Technology, India \\ Reviewed by: \\ Sunao Uchida, Waseda University, \\ Japan \\ V. Mohan Kumar, Sree Chitra Tirunal \\ Institute for Medical Sciences and \\ Technology, India \\ *Correspondence: \\ Bindu M. Kutty, Department of \\ Neurophysiology, National Institute of \\ Mental Health and Neurosciences, \\ Bangalore 560029, India. \\ e-mail: bindu.nimhans@gmail.com
}

Intense meditation practices help to achieve a harmony between body and mind. Meditation practices influence brain functions, induce various intrinsic neural plasticity events, modulate autonomic, metabolic, endocrine, and immune functions and thus mediate global regulatory changes in various behavioral states including sleep. This brief review focuses on the effect of meditation as a self regulatory phenomenon on sleep.

Keywords: meditation, sleep, brain functions, autonomic activity, endocrine functions, neural plasticity

\section{INTRODUCTION}

Meditation practices have been a life style practiced in India thousands of years ago. Proficient meditative practices help to integrate the brain functions, regulate various physiological mechanisms resulting in a state of mental and physical well being. Studies of long term transcendental meditation (TM) practitioners have shown that TM helped to achieve a state of "restful alertness" a state of deep physiological rest which was associated with periods of respiratory suspension without compensatory hyperventilation, decreased heart rate, heightened galvanic skin response along with enhanced wakefulness (Wallace, 1970). This restful alertness and hypometabolic state were believed to be the outcome of physiological and biochemical changes brought about by meditation practices (Young and Taylor, 1998).

\section{MEDITATION, BRAIN FUNCTIONS, AND SLEEP}

The effect of meditation on sleep was first reported by Mason et al. (1997) in practitioners of TM. The main objective was to evaluate the neurophysiological correlates of the higher states of consciousness during sleep. The study reported that the senior meditators spent more time in the slow wave sleep (SWS) with higher thetaalpha power with background delta activity, together with reduced electromyogram (EMG). The rapid eye movement (REM) sleep was also found to be enhanced. The distinct theta-alpha pattern observed during sleep was considered as an electrophysiological correlate of a stabilized state of higher consciousness in sleep. Further, the study opened up new avenues to explore the influence of meditation on sleep.

Studies by Sulekha et al. (2006) and Ravindra et al. (2010) demonstrated the differences in sleep architecture in practitioners of Vipassana meditation (mindfulness meditation). The sleep architecture of senior practitioners of Vipassana meditation was endowed with enhanced states of SWS and REM sleep compared to that of non-meditating control group. Vipassana meditators showed 17.95, 11.3, and $10.63 \%$ of SWS among younger ( $30-39$ years), middle ( $40-49$ years), and older (5060 years) age groups respectively. On the other hand, the corresponding non-meditating controls showed a significant reduction of SWS with increasing age, i.e., 11.29, 6.65, and 3.94\%. Vipassana meditators from all age groups showed more number of sleep cycles, indicating quality sleep. The study suggested that the older meditators could retain the sleep pattern of younger non-meditating controls. Aging is known to reduce the activity of the slow wave resonating mechanism either by actual loss of neurons or reduced activation of thalamo-cortical pathways (Feinberg et al., 1967; Mourtazaev et al., 1995) and also by reduced spindle generation during NREM sleep stage 2 (Nicolas et al., 2001). Vipassana meditation appears to preserve the SWS, suggesting that meditation could prevent the age associated changes in the slow wave generating mechanisms. Vipassana meditation also enhanced the REM sleep states. Meditation practices are reported to enhance the amplitude of gamma synchrony, strengthen the thalamocortical and cortico-cortical interactions (Lutz et al., 2004). These mechanisms brought about stronger network synchronization and altered the neural structure and functions (Lazar et al., 2005; Pagnoni and Cekic, 2007). Based on the above observations, the changes in sleep architecture in the Vipassana meditation practitioners could be attributed to the neural plasticity events associated with meditation.

\section{MEDITATION, AUTONOMIC ACTIVITY, AND SLEEP}

Changes in autonomic activity had been reported with respect to specific sleep states with predominant parasympathetic activity in SWS and sympathetic activity during REM sleep (Pivik et al., 1996; Otzenberger et al., 1997; Trinder et al., 2001; Pedemonte et al., 2005). Such sleep state dependent autonomic changes maintain the homeostasis during sleep. Aging alters autonomic flexibility leading to an overall increase in sympathetic activity along with reduced parasympathetic activity, thereby bringing about autonomic arousal and decrease in sleep quality. Reduced 
parasympathetic activity along with inefficient baroreflex mechanisms during REM sleep have been reported to cause unfavorable cardiac events (Somers et al., 1993; Ramaekers et al., 1999; Jones et al., 2003). Meditation practices help to bring about sympathovagal balance with parasympathetic predominance among experienced meditators and also in novice meditators with less practice (Wu and Lo, 2008; Zeidan et al., 2010). Vipassana meditation practices help to retain the flexibility of autonomic activity during different stages of sleep. Further, heart rate variability evaluation during REM sleep showed higher sympathetic activity in meditators than in controls. This higher sympathetic activity in meditators was effectively buffered by parasympathetic activity unlike the non-meditating controls (unpublished data). These studies have demonstrated a greater insight into the modulatory effect of meditation practices on autonomic functions during sleep. Meditation practices are associated with enhanced frontal midline theta activity (Aftanas and Golocheikine, 2001; Travis and Shear, 2010). The frontal midline theta activity originates from the anterior cingulate cortex and controls the parasympathetic activity (Tang et al., 2009). Vipassana meditation practices would have activated the anterior cingulate cortex and hence modulated the parasympathetic activity during sleep. These reports are suggestive of a positive modulatory role of meditation in sleep through autonomic functions.

\section{MEDITATION, MELATONIN, AND SLEEP}

Meditation practices were reported to regulate the hypothalamo pituitary adrenal (HPA) Axis and thereby the cortisol and catecholamine levels (Jevning et al., 1978a; Infante et al., 2001). Further, meditation techniques were also known to increase dehydroepiandrosterone (Glaser et al., 1992), anterior Pituitary hormones like growth hormone, thyroid stimulating hormone (TSH), prolactin (Jevning et al., 1978b; Werner et al., 1986; MacLean et al., 1997), and melatonin levels (Massion et al., 1995; Tooley et al., 2000).

Melatonin plays a vital role in the physiological regulation of sleep in both blind and normal individuals (Pandi-Perumal et al., 2006). Melatonin rhythm follows a raising and falling phase with corresponding alterations in sleep propensity (Dijk and Cajochen, 1997; Dijk et al., 1997). Melatonin exerts its hypnotic effect by acute inhibition of suprachiasmatic nucleus (von Gall et al., 2002) and also by facilitating hypothermic response through peripheral vasodilatation (Krauchi et al., 1997). Melatonin is widely used in the management of sleep rhythm disorders due to jetlag, shiftwork, and insomnia (Martinez and Lenz, 2010). In addition to its role in sleep, melatonin acts as an antioxidant and immunomodulator (Maestroni, 2001), oncostatic, antiaging agent, and helps in bringing sense of wellbeing (Armstrong and Redman, 1991; Reiter, 1995; Maestroni, 2001; Guerrero and Reiter, 2002; Pandi-Perumal et al., 2006). Aging attenuates the melatonin secretion (Sack et al., 1986) and hence affect the sleep quality in aged population.

Meditation practices are reported to enhance the melatonin levels (Tooley et al., 2000), the precursors of melatonin especially the serotonin (Bujatti and Riederer, 1976) and noradrenalin (Lang et al., 1979). Meditation increases melatonin concentration by slowing its hepatic metabolism or augmenting the synthesis in the pineal gland (Massion et al., 1995). Diurnal melatonin levels were found to be significantly high in Vipassana meditators (approximately $300 \mathrm{pg} \mathrm{ml}$ ) than non-meditating controls (65 pg ml; unpublished data). By considering the role of melatonin in sleep maintenance, it might be concluded that meditation practices enhance melatonin levels and hence quality of sleep.

\section{SLEEP AS AN AUTOREGULATORY, GLOBAL PHENOMENON}

Sleep is reported to be associated with reduced heart rate, blood pressure, respiratory rate, and rhythm, oxygen consumption, anxiety or arousal, and an overall decrease in basal metabolic levels leading to a hypometabolic state. This phenomenon of sleep induced hypometabolic state is a natural and spontaneous outcome necessary for biological survival (Young and Taylor, 1998). Imaging studies have shown that during NREM sleep the blood flow to areas associated with executive functions such as frontal and parietal cortex, thalamus, basal ganglia, and cerebellum has been reduced and bringing about the feeling of hypnogogic effect during sleep (Kajimura et al., 1999; Kjaer et al., 2002).

Meditation also brings a sustained hypometabolic state termed as relaxation response by Herbert Benson and helps in sleep initiation (Wallace et al., 1971). Similarly, meditation techniques help to regulate the blood flow to the executive regions of the brain during sleep (Lou et al., 1999). Meditation practices down regulate HPA axis reducing the stress, prolactin, TSH levels (Jevning et al., 1978a); bring about alterations in the intermediary metabolism favoring an anabolic state. Thus, meditation helps to maintain a wakeful hypometabolic state with parasympathetic predominance (Young and Taylor, 1998). Both the state and trait characteristics of meditation practices provide an advantage that it continually resets the metabolic functioning despite varying levels of stress. This internal metabolic resetting form the baseline trait characteristics necessary for all potential changes brought about by meditation practices. Further, meditative practices beneficially influence the cognitive, emotional, and behavioral aspects. Thus meditation is shown to have a greater potential to influence many physiological and behavioral states including sleep (Carlson et al., 2007; Ong et al., 2008; Sibinga et al., 2011).

It has been hypothesized that meditation practices activate prefrontal cortex, fronto-limbic, fronto-parietal neural networks and limbic and paralimbic cortices associated with sympathetic arousal. Meditation practices activate structures like insula, anterior cingulate, and hypothalamus and bring about autonomic and humoral changes (Newberg and Iversen, 2003). Meditation thus produces a continuum of global regulatory changes at various behavioral levels favoring quality sleep.

\section{CONCLUSION}

It is evident from the literature cited that practice of meditation brings about global changes. Many of these alterations in physiological functions have great similarities to the changes that are happening during sleep. It has been proposed that sleep is an autoregulatory global phenomenon (Kumar, 2010). It is also true that meditation influences sleep and its functions. It appears that various components of sleep generating mechanisms can be altered with meditation. Meditation, with its global effects on body and brain functions helps to establish a body and mind harmony. Thus meditation practices as an autoregulatory integrated global phenomenon, opens a wider scope for understanding the unique aspects of human sleep and consciousness. 


\section{REFERENCES}

Aftanas, L. I., and Golocheikine, S. A. (2001). Human anterior and frontal midline theta and lower alpha reflect emotionally positive state and internalized attention: high-resolution EEG investigation of meditation. Neurosci. Lett. 310, 57-60.

Armstrong, S. M., and Redman, J. R. (1991). Melatonin: a chronobiotic with anti-aging properties? Med. Hypotheses 34, 300-309.

Bujatti, M., and Riederer, P. (1976). Serotonin, noradrenaline, dopamine metabolites in transcendental meditation-technique. J. Neural Transm. 39, 257-267.

Carlson, L. E., Speca, M., Faris, P., and Patel, K. D. (2007). One year pre-post intervention follow-up of psychological, immune, endocrine and blood pressure outcomes of mindfulness-based stress reduction (MBSR) in breast and prostate cancer outpatients. Brain Behav. Immun. 21, 1038-1049.

Dijk, D. J., and Cajochen, C. (1997). Melatonin and the circadian regulation of sleep initiation, consolidation, structure, and the sleep EEG. J. Biol. Rhythms 12, 627-635.

Dijk, D. J., Shanahan, T. L., Duffy, J. F., Ronda, J. M., and Czeisler, C. A. (1997). Variation of electroencephalographic activity during non-rapid eye movement and rapid eye movement sleep with phase of circadian melatonin rhythm in humans. J. Physiol. (Lond.) 505(Pt 3), 851-858.

Feinberg, I., Koresko, R. L., and Heller, N. (1967). EEG sleep patterns as a function of normal and pathological aging in man. J. Psychiatr. Res. 5, 107-144.

Glaser, J. L., Brind, J. L., Vogelman, J. H., Eisner, M. J., Dillbeck, M. C., Wallace, R. K., Chopra, D., and Orentreich, N. (1992). Elevated serum dehydroepiandrosterone sulfate levels in practitioners of the transcendental meditation (TM) and TMSidhi programs. J. Behav. Med. 15, 327-341.

Guerrero, J. M., and Reiter, R. J. (2002). Melatonin-immune system relationships. Curr. Top. Med. Chem. 2, 167-179.

Infante, J. R., Torres-Avisbal, M., Pinel, P., Vallejo, J. A., Peran, F., Gonzalez, F., Contreras, P., Pacheco, C., Roldan, A., and Latre, J. M. (2001). Catecholamine levels in practitioners of the transcendental meditation technique. Physiol. Behav. 72, 141-146.

Jevning, R., Wilson, A. F., and Smith, W. R. (1978a). The transcendental meditation technique, adrenocortical activity, and implications for stress. Experientia 34, 618-619.

Jevning, R., Wilson, A. F., and VanderLaan, E. F. (1978b). Plasma prolactin and growth hormone during meditation. Psychosom. Med. 40,329-333.

Jones, P. P., Christou, D. D., Jordan, J., and Seals, D. R. (2003). Baroreflex buffering is reduced with age in healthy men. Circulation 107, 1770-1774.

Kajimura, N., Uchiyama, M., Takayama, Y., Uchida, S., Uema, T., Kato, M., Sekimoto, M., Watanabe, T., Nakajima, T., Horikoshi, S., Ogawa, K., Nishikawa, M., Hiroki, M., Kudo, Y., Matsuda, H., Okawa, M., and Takahashi, K. (1999). Activity of midbrain reticular formation and neocortex during the progression of human non-rapid eye movement sleep. J. Neurosci. 19, 10065-10073.

Kjaer, T. W., Law, I., Wiltschiotz, G., Paulson, O. B., and Madsen, P. L. (2002). Regional cerebral blood flow during light sleep - a H(2)(15)OPET study. J. Sleep Res. 11, 201-207.

Krauchi, K., Cajochen, C., Danilenko, K. V., and Wirz-Justice, A. (1997). The hypothermic effect of late evening melatonin does not block the phase delay induced by concurrent bright light in human subjects. Neurosci. Lett. 232, 57-61.

Kumar, V. M. (2010). Sleep is neither a passive nor an active phenomenon. Sleep Biol. Rhythms 8, 163-169.

Lang, R., Dehof, K., Meurer, K. A., and Kaufmann, W. (1979). Sympathetic activity and transcendental meditation. J. Neural Transm. 44, 117-135.

Lazar, S. W., Kerr, C. E., Wasserman, R. H., Gray, J. R., Greve, D. N., Treadway, M. T., McGarvey, M., Quinn, B. T., Dusek, J. A., Benson, H., Rauch, S. L., Moore, C. I., and Fischl, B. (2005). Meditation experience is associated with increased cortical thickness. Neuroreport 16, 1893-1897.

Lou, H. C., Kjaer, T. W., Friberg, L., Wildschiodtz, G., Holm, S., and Nowak, M. (1999). A 15O-H2O PET study of meditation and the resting state of normal consciousness. Hum. Brain Mapp. 7, 98-105.

Lutz, A., Greischar, L. L., Rawlings, N. B., Ricard, M., and Davidson, R. J. (2004). Long-term meditators self-induce high-amplitude gamma synchrony during mental practice. Proc. Natl. Acad. Sci. U.S.A. 101, 16369-16373.

MacLean, C. R., Walton, K. G., Wenneberg, S. R., Levitsky, D. K., Mandarino, J. P., Waziri, R., Hillis, S. L., and Schneider, R. H. (1997). Effects of the transcendental meditation program on adaptive mechanisms: changes in hormone levels and responses to stress after 4 months of practice. Psychoneuroendocrinology 22, 277-295.

Maestroni, G. J. (2001). The immunotherapeutic potential of melatonin. Expert Opin. Investig. Drugs 10, 467-476.

Martinez, D., and Lenz, M. C. (2010). Circadian rhythm sleep disorders. Indian J. Med. Res. 131, 141-149.

Mason, L. I., Alexander, C. N., Travis, F. T., Marsh, G., Orme-Johnson, D. W., Gackenbach, J., Mason, D. C., Rainforth, M., and Walton, K. G. (1997). Electrophysiological correlates of higher states of consciousness during sleep in long-term practitioners of the transcendental meditation program. Sleep 20, 102-110.

Massion, A. O., Teas, J., Hebert, J. R. Wertheimer, M. D., and Kabat-Zinn, J. (1995). Meditation, melatonin and breast/prostate cancer: hypothesis and preliminary data. Med. Hypotheses 44, 39-46.

Mourtazaev, M. S., Kemp, B., Zwinderman, A. H., and Kamphuisen, H. A. (1995). Age and gender affect different characteristics of slow waves in the sleep EEG. Sleep 18, 557-564.

Newberg, A. B., and Iversen, J. (2003). The neural basis of the complex mental task of meditation: neurotransmitter and neurochemical considerations. Med. Hypotheses 61, 282-291.

Nicolas, A., Petit, D., Rompre, S., and Montplaisir, J. (2001). Sleep spindle characteristics in healthy subjects of different age groups. Clin Neurophysiol 112, 521-527.

Ong, J. C., Shapiro, S. L., and Manber, R. (2008). Combining mindfulness meditation with cognitive-behavior therapy for insomnia: a treatmentdevelopment study. Behav. Ther. 39, 171-182.

Otzenberger, H., Simon, C., Gronfier, C., and Brandenberger, G. (1997). Temporal relationship between dynamic heart rate variability and electroencephalographic activity during sleep in man. Neurosci. Lett. 229, 173-176.

Pagnoni, G., and Cekic, M. (2007). Age effects on gray matter volume and attentional performance in Zen meditation. Neurobiol. Aging 28, 1623-1627.

Pandi-Perumal, S. R., Srinivasan, V., Maestroni, G. J., Cardinali, D. P., Poeggeler, B., and Hardeland, R. (2006). Melatonin: nature's most versatile biological signal? FEBS J. 273, 2813-2838.

Pedemonte, M., Rodriguez-Alvez, A., and Velluti, R. A. (2005). Electroen- cephalographic frequencies associated with heart changes in RR interval variability during paradoxical sleep. Auton. Neurosci. 123, 82-86.

Pivik, R. T., Busby, K. A., Gill, E., Hunter, P., and Nevins, R. (1996). Heart rate variations during sleep in preadolescents. Sleep 19, 117-135.

Ramaekers, D., Ector, H., and Aubert, A. E. (1999). The influence of age and gender on heart rate variability (HRV). J. Am. Coll. Cardiol. 33, 900-902.

Ravindra, P. N., Sulekha, S., Sathyaprabha, T. N., Pradhan, N., Raju, T. R., and Kutty, B. M. (2010). Practitioners of vipassana meditation exhibit enhanced slow wave sleep and REM sleep states across different age groups. Sleep Biol. Rhythms 8, 34-41.

Reiter, R. J. (1995). Oxygen radical detoxification processes during aging: the functional importance of melatonin. Aging (Milano) 7, 340-351.

Sack, R. L., Lewy, A. J., Erb, D. L., Vollmer, W. M., and Singer, C. M. (1986). Human melatonin production decreases with age. J. Pineal Res. 3, 379-388.

Sibinga, E. M., Kerrigan, D., Stewart, M., Johnson, K., Magyari, T., and Ellen, J. M. (2011). Mindfulness-based stress reduction for urban youth. J. Altern. Complement. Med. 17, 213-218.

Somers, V. K., Dyken, M. E., Mark, A. L., and Abboud, F. M. (1993). Sympathetic-nerve activity during sleep in normal subjects. N. Engl. J. Med. 328, 303-307.

Sulekha, S., Thennarasu, K. Vedamurthachar, A., Raju, T. R., and Kutty, B. M. (2006). Evaluation of sleep architecture in practitioners of Sudarshan Kriya yoga and Vipassana meditation. Sleep Biol. Rhythms 4, 207-214.

Tang, Y. Y., Ma, Y., Fan, Y., Feng, H., Wang, J., Feng, S., Lu, Q., Hu, B., Lin, Y., Li, J., Zhang, Y., Wang, Y., Zhou, L., and Fan, M. (2009). Central and autonomic nervous system interaction is altered by short-term meditation. Proc. Natl. Acad. Sci. U.S.A. 106, 8865-8870.

Tooley, G. A., Armstrong, S. M., Norman, T. R., and Sali, A. (2000). Acute increases in night-time plasma melatonin levels following a period of meditation. Biol. Psychol. 53, 69-78.

Travis, F., and Shear, J. (2010). Focused attention, open monitoring and automatic self-transcending: categories to organize meditations from Vedic, Buddhist and Chinese traditions. Conscious. Cogn. 19, 1110-1118. 
Trinder, J., Kleiman, J., Carrington, M., Smith, S., Breen, S., Tan, N., and Kim, Y. (2001). Autonomic activity during human sleep as a function of time and sleep stage. J. Sleep Res. 10, 253-264.

von Gall, C., Stehle, J. H., and Weaver, D. R. (2002). Mammalian melatonin receptors: molecular biology and signal transduction. Cell Tissue Res. 309, 151-162.

Wallace, R. K. (1970). Physiological effects of transcendental meditation. Rev. Bras. Med. 27, 397-401.

Wallace, R. K., Benson, H., and Wilson, A. F. (1971). A wakeful hypometabolic physiologic state. Am. J. Physiol. 221, 795-799.

Werner, O. R., Wallace, R. K., Charles, B., Janssen, G., Stryker, T., and Chalmers, R. A. (1986). Longterm endocrinologic changes in subjects practicing the transcendental meditation and TM-Sidhi program. Psychosom. Med. 48, 59-66.

Wu, S. D., and Lo, P. C. (2008). Inwardattention meditation increases parasympathetic activity: a study based on heart rate variability. Biomed. Res. 29, 245-250.

Young, J. D., and Taylor, E. (1998). Meditation as a voluntary hypometabolic state of biological estivation. News Physiol. Sci. 13, 149-153.

Zeidan, F., Johnson, S. K., Gordon, N. S., and Goolkasian, P. (2010). Effects of brief and sham mindfulness meditation on mood and cardiovascular variables. J. Altern. Complement. Med. 16, 867-873.

Conflict of Interest Statement: The authors declare that the research was conducted in the absence of any commercial or financial relationships that could be construed as a potential conflict of interest.

Received: 21 March 2012; paper pending published: 26 March 2012; accepted: 27
March 2012; published online: 18 April 2012.

Citation: Nagendra RP, Maruthai $N$ and Kutty BM (2012) Meditation and its regulatory role on sleep. Front. Neur. 3:54. doi: 10.3389/fneur.2012.00054

This article was submitted to Frontiers in Sleep and Chronobiology, a specialty of Frontiers in Neurology.

Copyright (c) 2012 Nagendra, Maruthai and Kutty. This is an open-access article distributed under the terms of the Creative Commons Attribution Non Commercial License, which permits noncommercial use, distribution, and reproduction in other forums, provided the original authors and source are credited. 